\title{
Adapting DESGW Single Epoch Image Differencing to the LSST Software Stack
}

\author{
Nathan Garrett, Dark Energy Survey | Supervisor - Ken Herner
}

\section{Old Pipeline, New Application}

As gravitational wave detections become an ever-increasing part of cosmological research, the Gravitational Waves Group of the Dark Energy Survey (DESGW) seeks to match and interpret optical observations for these short-lived, transient events. ${ }^{[1]}$ To provide timely analysis for each follow up, DESGW has developed a custom Single Epoch Differencing Pipeline for processing optical data obtained from the Dark Energy Camera (DECam). ${ }^{[2]}$ While effective, the pipeline is a custom design and thus requires extra maintenance and specialized knowledge to operate and modify at a time when new projects are taking precedence.

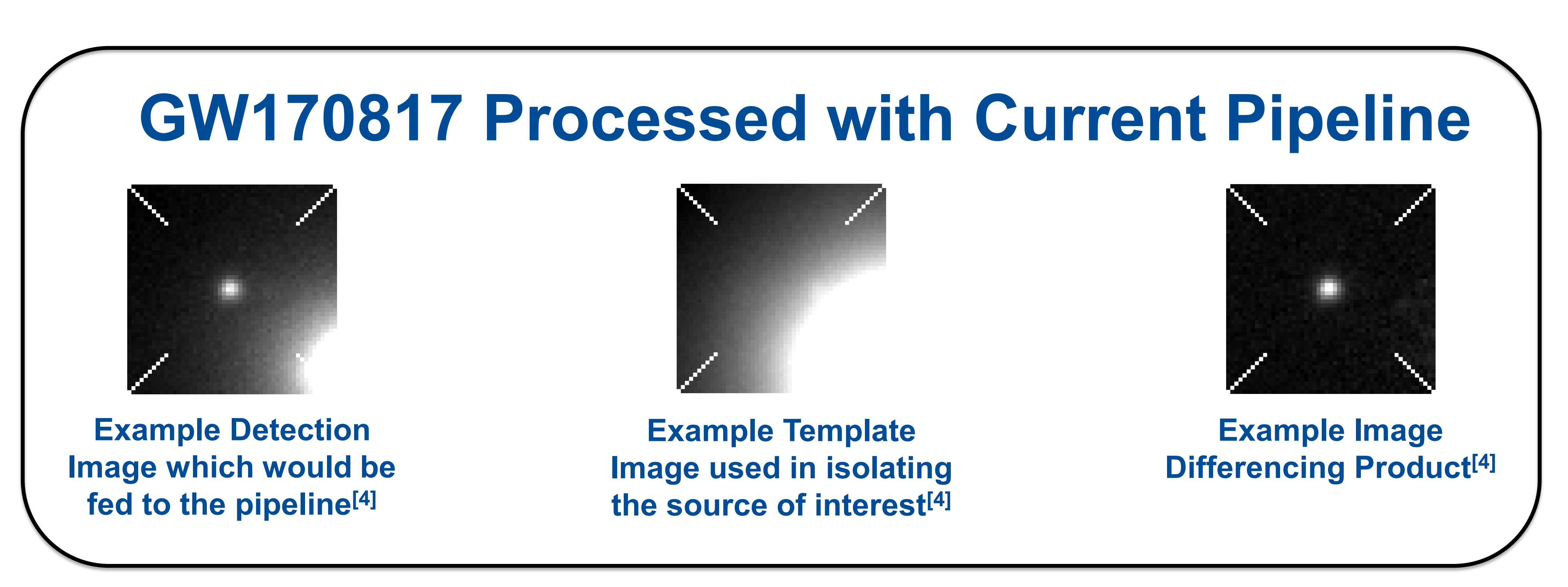

Current Image

\section{Pipeline:}

Flowchart adapted from
Herner et al, 2020

New Pipeline

Using LSST Stack:
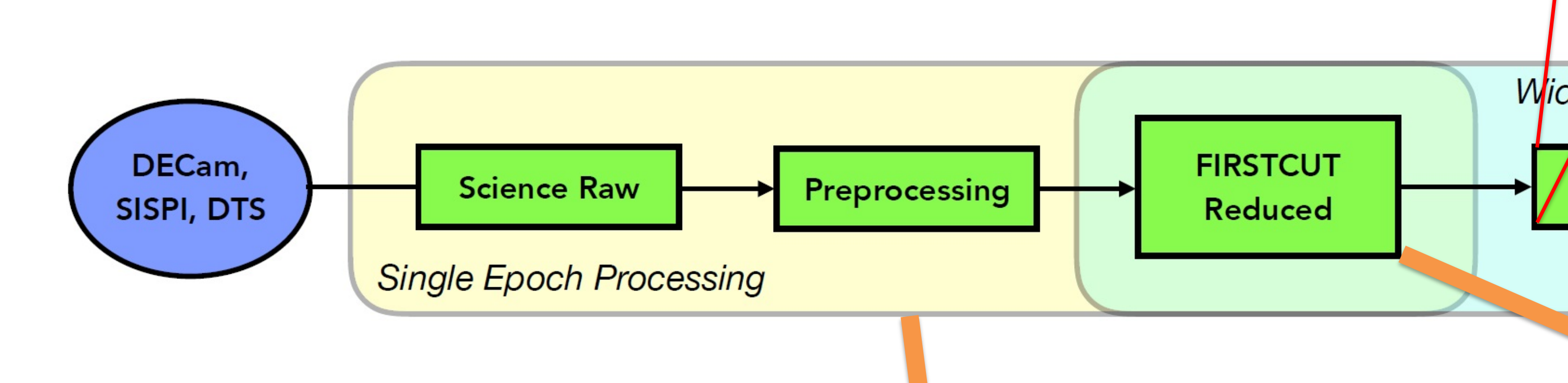
Raw data is ingested and cataloged into by the pipeline tasks during processing Calibrations and reference cataloss
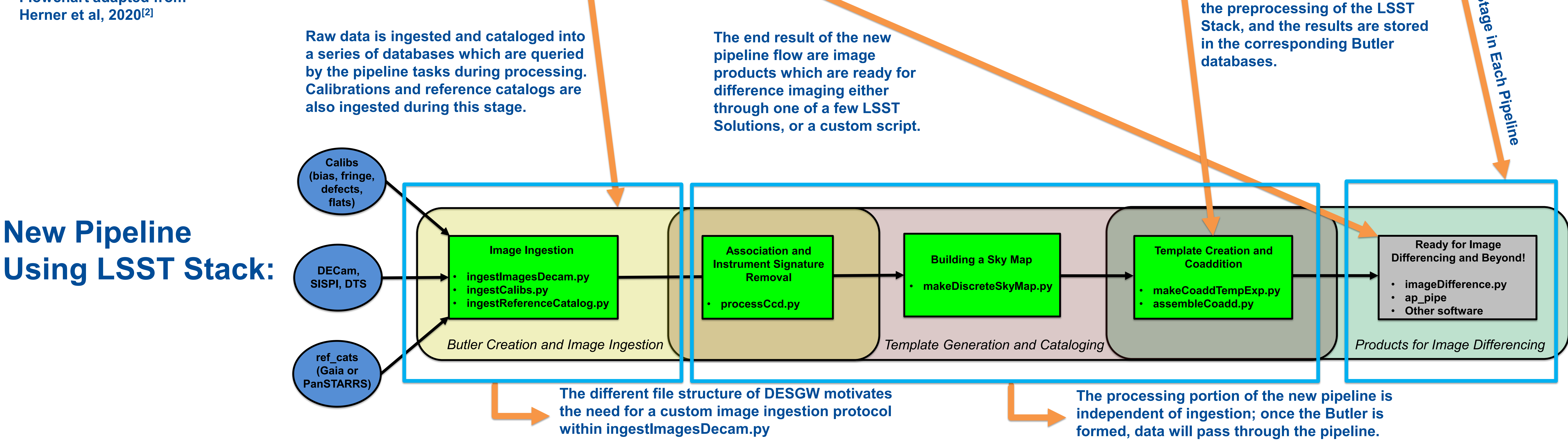

\section{LSST Software Stack}

The image processing pipeline produced for the Legacy Survey of Space and Time (LSST) provides difference image analysis functionality for a wide array of large telescopes, including DECam. ${ }^{[3]}$ Repurposing DECam specific elements of the LSST Software Stack

has potential to provide balance between efficient maintenance and effective, accurate analysis.

\section{Advantages}

- Open source platform with extensive, active community forum

- Support for many large survey telescopes, including existing limited support for DECam applications

- Butler database for registering data and pipeline scripts simplify data consolidation and image processing

\section{Remaining Challenges}

- Expected formatting differs from DESGW Data

- Pipeline requires specific metadata items

- Pipeline wholly dependent on stackcreated Butler Databases for incorporating data

- Butler dependence leads to failure when data doesn't ingest

\section{References}

1. Dark Energy Survey, The Dark Energy Survey, https./Twww.darkenergysurvey.org/ (Accessed July 2020).

2. Herner, K. et al.. "Optical follow-up of gravitational wave triggers with DECam during the firsttwo LIGO/VIRGO observing runs." arXiv:2001.06551 (2020).

3. LSST, The LSST Science Pipelines, https://pipelines.Isst.io/ (Accessed July 2020). Herner, K., "GW170817: Discovery of the Optical Counterpart of a Neutron Star Merger with DECam." Fermilab Computing Techniques Seminar (2018).

This work was supported in part by the U.S. Department of Energy, Office of Science, Office of Workforce Development for Teachers and Scientists (WDTS) under the Science Undergraduate Laboratory Internships
Program (SULI). 